LA-14404

Approved for public release;

distribution is unlimited.

\title{
Alignment of Leading-Edge and Peak-Picking \\ Time of Arrival Methods to Obtain Accurate Source Locations
}


This work was sponsored by Science Applications International Corporation, Melbourne, FL, and Los Alamos National Laboratory's Environmental Science Division and Nuclear Nonproliferation Division, Los Alamos, NM.

Edited by Mable Amador, Group IRM-CAS

Photocomposition by Deidre' A. Plumlee, Group IRM-CAS

Los Alamos National Laboratory, an affirmative action/ equal opportunity employer, is operated by Los Alamos National Security, LLC, for the National Nuclear Security Administration of the U.S. Department of Energy under contract DE-AC52-06NA25396.

This report was prepared as an account of work sponsored by an agency of the U.S. Government. Neither Los Alamos National Security, LLC, the U.S. Government nor any agency thereof, nor any of their employees make any warranty, express or implied, or assume any legal liability or responsibility for the accuracy, completeness, or usefulness of any information, apparatus, product, or process disclosed, or represent that its use would not infringe privately owned rights. Reference herein to any specific commercial product, process, or service by trade name, trademark, manufacturer, or otherwise does not necessarily constitute or imply its endorsement, recommendation, or favoring by Los Alamos National Security, LLC, the U.S. Government, or any agency thereof. The views and opinions of authors expressed herein do not necessarily state or reflect those of Los Alamos National Security, LLC, the U.S. Government, or any agency thereof. Los Alamos National Laboratory strongly supports academic freedom and a researcher's right to publish; as an institution, however, the Laboratory does not endorse the viewpoint of a publication or guarantee its technical correctness. 
LA-14404

Issued: August 2009

\section{Alignment of Leading-Edge and Peak-Picking \\ Time of Arrival Methods to Obtain Accurate \\ Source Locations}

R. Roussel-Dupré*

Eugene Symbalisty

Craig Fox**

Orion Vanderlinde**

* Consultant at Los Alamos National Laboratory, Computational Earth Sciences, Sci Tech Solutions, Santa Fe, NM 87506.

** Science Applications International Corporation, Melbourne, FL. 
This page intentionally left blank. 


\section{Table of Contents}

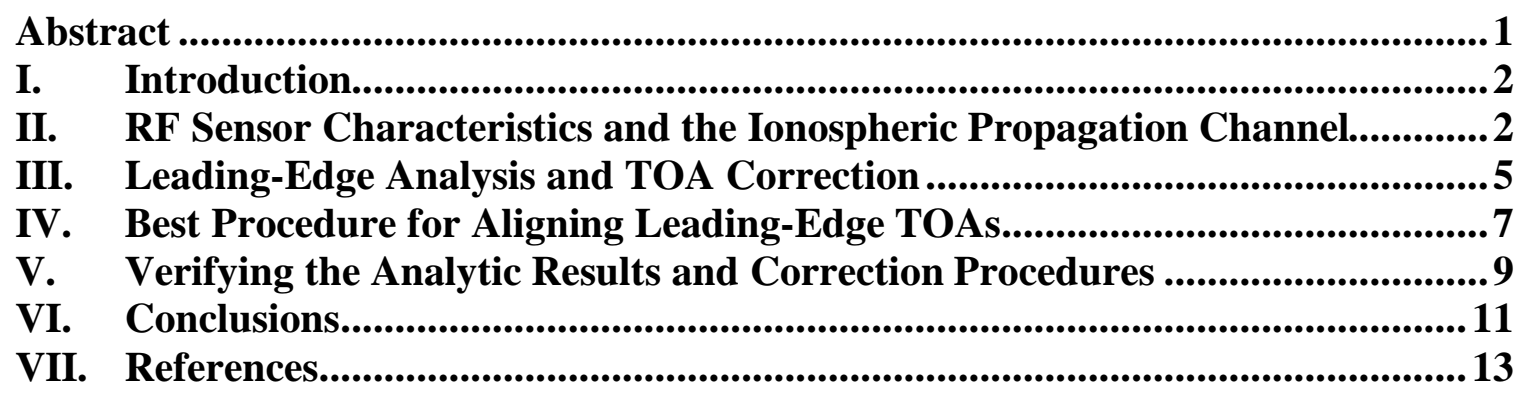


This page intentionally left blank. 


\title{
Alignment of Leading-Edge and Peak-Picking Time of Arrival Methods to Obtain Accurate Source Locations
}

by

\author{
R. Roussel-Dupré, E. Symbalisty, C. Fox, and O. Vanderlinde
}

\begin{abstract}
The location of a radiating source can be determined by time-tagging the arrival of the radiated signal at a network of spatially distributed sensors. The accuracy of this approach depends strongly on the particular time-tagging algorithm employed at each of the sensors. If different techniques are used across the network, then the time tags must be referenced to a common fiducial for maximum location accuracy. In this report we derive the time corrections needed to temporally align leading-edge, time-tagging techniques with peak-picking algorithms. We focus on broadband radio frequency (RF) sources, an ionospheric propagation channel, and narrowband receivers, but the final results can be generalized to apply to any source, propagation environment, and sensor. Our analytic results are checked against numerical simulations for a number of representative cases and agree with the specific leading-edge algorithm studied independently by Kim and Eng (1995) and Pongratz (2005 and 2007).
\end{abstract}




\section{Introduction}

Determination of a source's location from signal measurements obtained with spatially distributed sensors can be accomplished by time-tagging the arrival of the signal at each of the sensors. The location of the source is deduced by combining the measured arrival times at each sensor with the known location of the sensors. The accuracy of this analysis depends strongly on the time-tagging technique, on the signal-to-noise ratio, on the spatial diversity of the sensors relative to the source, on identifying a unique signal feature that can be tagged at all the sensors, and on avoiding temporal features in the signal that are produced by other means, such as a delayed secondary source, reflections, or "multipathing" caused by propagation of the signal through a structured environment.

Two of the most commonly used time-tagging techniques involve the leading edge of the signal or the peak. Each has its advantages and disadvantages. In the case of a distributed network of sensors that employs a mixture of time-of-arrival (TOA) techniques, it is necessary to reference the disparate arrival times to a common fiducial in order to obtain as accurate a source location as possible.

The formulae necessary to convert the leading-edge arrival times to peak arrival times for application to a mixed network that uses one or the other TOA technique at different sensors are derived. We then discuss the appropriate procedure for aligning the leadingedge TOAs for three signal regimes that involve only one impulse or an initial pulse followed by several delayed, secondary pulses. Numerical simulations that verify the analytic results in the case of a single impulse arriving at the detectors are described. Our analysis is focused on a constellation of satellite-based sensors that measure the radio frequency (RF) signal from impulsive sources in the Earth's atmosphere such as lightning. Although only broadband RF sources, an ionospheric propagation channel, and narrowband receivers are invoked in this analysis, the technique and final results can be generalized to apply to any source, propagation environment, and sensor.

The analytic derivations presented in this report were obtained before our reading of the reports by Pongratz (2005 and 2007) and by Kim and Eng (1995), who considered a specific case related to leading-edge TOAs and peak-picking TOAs.

\section{RF Sensor Characteristics and the Ionospheric Propagation Channel}

In this study, we focus our attention on broadband RF signals measured by narrowband RF sensors. The intervening propagation channel between the source and the sensor is assumed to be dispersive only. For most geometries of interest, this description applies to the Very High Frequency (VHF) components of a broadband signal that propagates through the Earth's ionosphere. Because we are primarily interested in the temporal signature of the signal for time-tagging purposes, what matters is the impulse response of the RF receiver convolved against that of the intervening propagation medium along a ray path from the source to the sensor.

The RF receiver is assumed to consist in part of a Surface Acoustic Wave (SAW) filter with a Gaussian shape [Transfer function, $\mathrm{T}_{\mathrm{f}}(\mathrm{f})$ ] in the Fourier domain and a 
corresponding Gaussian impulse response $\left[\mathrm{I}_{\mathrm{f}}(\mathrm{t})\right]$ in the time domain. Mathematically, we have the following (Eq. 1):

$T_{f}(f)=\left[e^{\frac{-\left(f-f_{0}\right)^{2}}{B^{2}}}+e^{\frac{-\left(f+f_{0}\right)^{2}}{B^{2}}}\right] e^{2 \pi \text { if } \tau_{f}}$

and

$\mathrm{I}_{\mathrm{f}}(\mathrm{t})=2 \mathrm{~B} \sqrt{\pi} \mathrm{e}^{-\pi^{2} \mathrm{~B}^{2}\left(\mathrm{t}-\tau_{\mathrm{f}}\right)^{2}} \cos \left[2 \pi \mathrm{f}_{0}\left(\mathrm{t}-\tau_{\mathrm{f}}\right)\right]$

where $f_{0}$ is the central frequency of the filter, $B$ is the $1 /$ e bandwidth, and $\tau_{f}$ is the group delay of the filter. The full width at half maximum of the filter can be written as follows (Eq. 2):

$\mathrm{B}_{\mathrm{FWHM}}=2 \sqrt{\ln 2} \mathrm{~B}$

The group delay of SAW filters depends on the bandwidth and is given by the following:

$\tau_{\mathrm{f}}=\frac{16 \sqrt{\ln 2}}{\pi \mathrm{B}_{\mathrm{FWHM}}}$

When convolved against the impulse response of the ionosphere, the transionospheric signal through a Gaussian filter can be written (see Massey, 1993) as follows: 


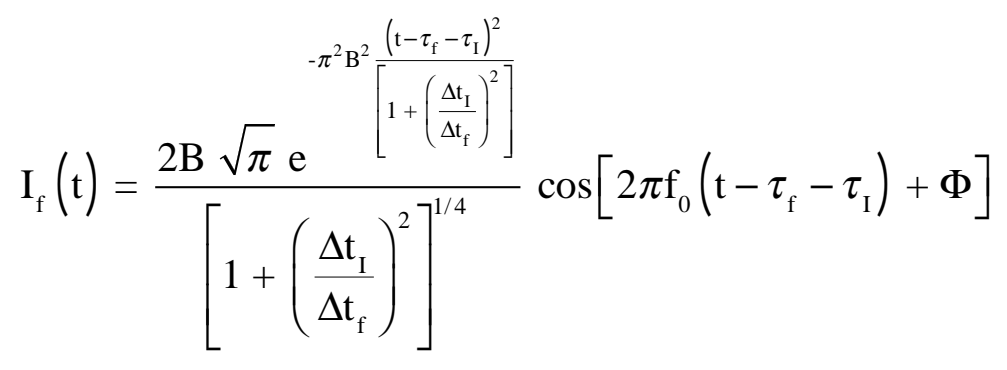

where

$$
\begin{gathered}
\tau_{\mathrm{I}}=\frac{8.43 \times 10^{3} \mathrm{TEC}}{2 \pi\left(\mathrm{f}_{0} \pm \mathrm{f}_{\mathrm{c}} \cos \beta\right)^{2}} \\
\Delta \mathrm{t}_{\mathrm{I}}=\frac{2 \tau_{\mathrm{I}} \sqrt{2 \pi / 8 \ln 2} \mathrm{~B}_{\mathrm{FWHM}}}{\left(\mathrm{f}_{0} \pm \mathrm{f}_{\mathrm{c}} \cos \beta\right)}
\end{gathered}
$$

$$
\Delta \mathrm{t}_{\mathrm{f}}=\frac{2 \sqrt{\ln 2}}{\sqrt{\pi} \mathrm{B}_{\mathrm{FWHM}}}
$$

$$
\Phi=\frac{1}{2} \tan ^{-1}\left(\frac{\Delta \mathrm{t}_{\mathrm{I}}}{\Delta \mathrm{t}_{\mathrm{f}}}\right)-\pi^{2} \mathrm{~B}^{2} \frac{\left(\mathrm{t}-\tau_{\mathrm{f}}-\tau_{\mathrm{I}}\right)^{2} \Delta \mathrm{t}_{\mathrm{I}}}{\left[1+\left(\frac{\Delta \mathrm{t}_{\mathrm{I}}}{\Delta \mathrm{t}_{\mathrm{f}}}\right)^{2}\right] \Delta \mathrm{t}_{\mathrm{f}}}
$$

and where TEC is the total electron content along the slant path from the source to the receiver in units of $10^{16} \mathrm{~m}^{-2}, \mathrm{f}_{\mathrm{c}}$ is the electron cyclotron frequency in the presence of the geomagnetic field, $\beta$ is the angle between the line-of-sight from the source to the receiver and the geomagnetic field. We have assumed that $\mathrm{B} / \mathrm{f}_{0} \ll 1$, all times are in microseconds, and all frequencies are in MHz. In the results of Eq. $4, \tau_{\mathrm{I}}$ is recognized to be the group delay of the ionosphere at frequency $\mathrm{f}_{0}, \Delta \mathrm{t}_{\mathrm{I}}$ is the ionospheric dispersion time across the filter, and $\Delta \mathrm{t}_{\mathrm{f}}$ is the filter response time. Note that $\mathrm{B}_{\mathrm{FWHM}}$ is the bandwidth of the receiver in amplitude (not the $3 \mathrm{~dB}$ bandwidth). We have added the effect of the geomagnetic field in our model of ionospheric propagation as it serves to split the incident electromagnetic pulse into two modes (ordinary, plus sign, and extraordinary, minus sign) with different propagation speeds and therefore different time delays, as can be seen in Eq. 4. The impact on time-tagging can be significant when both modes are accepted by the receiver as a result of the beating of the two modes and the corresponding 
distortion of the recorded signal. We do not treat this issue in this report but include the magnetic correction as a reminder of its role and potential impact.

\section{Leading-Edge Analysis and TOA Correction}

The primary advantage of this time-tagging method is that it uses the early part of the signal (before the arrival of the peak). In this way, the confusion in signal arrival times introduced by secondary sources, reflections, and multipathing are avoided. The primary disadvantage is that the signal amplitudes and associated times used in determining a TOA are less than the peak and are therefore subject to uncertainties introduced by noise. The impact of the noise depends on the values selected along the leading edge.

Let $\mathrm{w}_{1}$ equal the amplitude of the signal, measured relative to the peak, that arrives at $t_{1}$ and similarly for $w_{2}$, which arrives at $t_{2}$. Let $t_{p}$ equal the arrival time of the peak signal. We can define the arrival time of the impulse (assumed to be a delta function in time that hits the filter at the arrival time) by fitting a straight line to these two points and extrapolating the line to zero amplitude (i.e., when the impulse first arrives and elicits a response from the filter) with the following result (Eq. 5):

$\operatorname{TOA}=\frac{-\mathrm{w}_{1}\left(\mathrm{t}_{2}-\mathrm{t}_{1}\right)}{\mathrm{w}_{2}-\mathrm{w}_{1}}+\mathrm{t}_{1}$

Note that all times are measured after envelope detection. The times $t_{2}$ and $t_{1}$ can be related to the time $t_{p}$ at which the peak amplitude arrives; using Eq. 4, we have the following (Eq. 6): 


$$
\mathrm{t}_{\mathrm{p}}=\tau_{\mathrm{f}}+\tau_{\mathrm{I}}
$$

$\mathrm{t}_{1}=\mathrm{t}_{\mathrm{p}}-\sqrt{\ln \left(1 / \mathrm{w}_{1}\right)} \frac{\left[1+\left(\frac{\Delta \mathrm{t}_{\mathrm{I}}}{\Delta \mathrm{t}_{\mathrm{f}}}\right)^{2}\right]^{1 / 2}}{\pi \mathrm{B}}$

$\mathrm{t}_{2}=\mathrm{t}_{\mathrm{p}}-\sqrt{\ln \left(1 / \mathrm{w}_{2}\right)} \frac{\left[1+\left(\frac{\Delta \mathrm{t}_{\mathrm{I}}}{\Delta \mathrm{t}_{\mathrm{f}}}\right)^{2}\right]^{1 / 2}}{\pi \mathrm{B}}$

and

$\mathrm{TOA}=\mathrm{t}_{\mathrm{p}}-\left[\frac{\mathrm{w}_{2} \sqrt{\ln \left(1 / \mathrm{w}_{1}\right)}-\mathrm{w}_{1} \sqrt{\ln \left(1 / \mathrm{w}_{2}\right)}}{\mathrm{w}_{2}-\mathrm{w}_{1}}\right] \frac{\left[1+\left(\frac{\Delta \mathrm{t}_{\mathrm{I}}}{\Delta \mathrm{t}_{\mathrm{f}}}\right)^{2}\right]^{1 / 2}}{\pi \mathrm{B}}$

These results provide a means of relating the derived TOA for each frequency channel to the signal peak arrival time by adding a correction term that depends on the two leading-edge amplitudes multiplied by the total 1/e temporal width of the detected signal (see Eq. 4). The result is Eq. 7 ,

$\Delta \mathrm{t}_{\text {total }}=\frac{\left[1+\left(\frac{\Delta \mathrm{t}_{\mathrm{I}}}{\Delta \mathrm{t}_{\mathrm{f}}}\right)^{2}\right]^{1 / 2}}{\pi \mathrm{B}}$.

A similar result was found by Kim and Eng (1995) for the specific case of $\mathrm{w}_{1}=1 / 3$ and $\mathrm{w}_{2}=2 / 3$. The total temporal width as discussed thus far includes both the filter response time and the ionospheric dispersion across the filter. If an accurate measurement of the width can be obtained, then it is possible to correct the leading edge TOA for each channel and proceed with the usual difference TOA (DTOA) analysis to remove ionospheric effects and obtain the infinite frequency arrival time for application in a mixed network geolocation determination. We emphasize that when the measured total temporal width is used in Eq. (6), the leading edge TOA is also corrected for any noise broadening or narrowing effects (see Pongratz, 2005). 


\section{Best Procedure for Aligning Leading-Edge TOAs}

Clearly, any attempt to align the leading-edge TOAs to peak TOAs will compromise the advantages associated with using the leading edge of the signal for time-tagging. The results obtained in the previous section indicate that TOA uncertainties associated with the late arrival of secondary pulses would show up primarily through distortions introduced in the temporal width of the signal. This insight leads us to separate the potential signals into three regimes.

The first occurs when no secondary signals arrive at the detector. In this case, the procedure of correcting the leading-edge TOAs for pulse-width effects (like noise broadening) followed by the infinite frequency time of arrival determination (removal of ionospheric effects) should yield the best results. A table lookup developed from simulations should also produce similar results. The second regime deals with the rapid arrival of additional impulsive signals following the signal of interest so that the leading edge of the first signal is now distorted. In this case, as in the first regime, the total width of the signal can be used to correct the leading-edge TOAs in a meaningful way that aligns them with peak TOAs that would also be affected by the arrival of all signals and not just the first. For this regime, the time delay of the follow-on signals is less than the $1 / \mathrm{e}$ width of the signal, i.e., $\mathrm{t}_{\text {delay }}<\Delta \mathrm{t}_{\text {total }}$. Note that noise broadening or narrowing of the signal is also included in this regime. In this case, as in the first regime, our analysis proceeds as follows. First, the leading-edge TOA for each channel is corrected so that

$$
\mathrm{TOA}_{\text {corr }}=\mathrm{TOA}_{\text {meas }}+\left[\frac{\mathrm{w}_{2} \sqrt{\ln \left(1 / \mathrm{w}_{1}\right)}-\mathrm{w}_{1} \sqrt{\ln \left(1 / \mathrm{w}_{2}\right)}}{\mathrm{w}_{2}-\mathrm{w}_{1}}\right] \Delta \mathrm{t}_{\text {total }} .
$$

The total pulse width $\Delta \mathrm{t}_{\text {total }}$ can be determined by fitting a parabola to the log power of the signal. Then the ionospheric delay is determined either by a linear fit to the corrected TOAs vs. $1 / \mathrm{f}_{0}^{2}$ (ignoring geomagnetic field effects) for all channels or by using

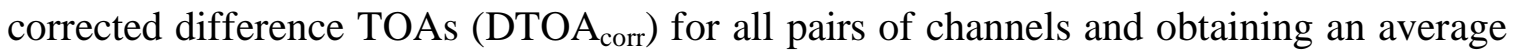
infinite frequency TOA, $\mathrm{t}_{0}$, in the usual way. The latter should then be corrected for the relative filter group delays of the leading-edge and peak-picking stations for use in a geolocation calculation. We thus have Eq. 9,

$$
\mathrm{t}_{\text {corr,LE }}=\mathrm{t}_{0, \mathrm{LE}}+\tau_{\mathrm{f}, \text { peak }}-\tau_{\mathrm{f}, \mathrm{LE}} .
$$

The third regime is defined by signals that arrive so that $t_{\text {delay }}>\Delta t_{\text {total }}$, assuming that the amplitudes of the secondary signals are less than or not much greater than the first impulse. In this case, the leading edge of the detected signal is undistorted and associated solely with the arrival of the signal of interest. Any attempt to use the signal width to correct the leading-edge TOAs would be subject to substantial errors introduced by 
secondary pulses. Of course, the success of both time-tagging techniques (leading edge and peak picking) depends on our ability to identify the first peak correctly. In this regime, our analysis proceeds as follows. First, note that for a leading-edge analysis, the TOA has a temporal width dependence that is TEC dependent, i.e., $\Delta \mathrm{t}_{\mathrm{I}}$ in Eq. 6 . In order to determine the infinite frequency TOA, $t_{0}$, we recast Eq. 6 with the help of Eq. 4 as follows:

$\operatorname{TOA}=\tau_{\mathrm{f}}+\mathrm{a}_{0} \operatorname{TEC}\left(\frac{1}{\mathrm{f}_{0}^{2}}\right)-\frac{\mathrm{w}}{\pi \mathrm{B}}\left[1+\frac{\mathrm{a}_{0}^{2} \mathrm{a}_{1}^{2} \mathrm{TEC}^{2}}{\Delta \mathrm{t}_{\mathrm{f}}^{2}}\left(\frac{1}{\mathrm{f}_{0}^{6}}\right)\right]^{1 / 2}$

where

$\mathrm{a}_{0}=\frac{8.43 \times 10^{3}}{2 \pi}, \mathrm{a}_{1}=2 \sqrt{2 \pi / 8 \ln 2} \mathrm{~B}_{\mathrm{FWHM}}$,

and

$\mathrm{w}=\left[\frac{\mathrm{w}_{2} \sqrt{\ln \left(1 / \mathrm{w}_{1}\right)}-\mathrm{w}_{1} \sqrt{\ln \left(1 / \mathrm{w}_{2}\right)}}{\mathrm{w}_{2}-\mathrm{w}_{1}}\right]$.

Eq. (10) indicates that we can write the measured TOAs as a function of frequency, as follows,

$\mathrm{TOA}-\tau_{\mathrm{f}}=\mathrm{a}+\mathrm{bX}-\frac{\mathrm{w}}{\pi \mathrm{B}}\left(1+\mathrm{c} \mathrm{X}^{3}\right)^{1 / 2}$

where

$\mathrm{X}=\frac{1}{\mathrm{f}_{0}^{2}}$ and $\mathrm{a}, \mathrm{b}$, and $\mathrm{c}$ are unknown coefficients.

A least-squares fit of the TOAs to the functional form given by Eq. (11) allows us to calculate the infinite frequency TOA, $\mathrm{t}_{0, \mathrm{LE}}($ at $\mathrm{X}=0$ ), and the TEC from the corresponding coefficients provided in Eq. (10). The three unknown coefficients require a minimum of four frequency channels using an iterative nonlinear least-squares fitting routine. The aligned, leading-edge, infinite-frequency arrival time is then given by 


$$
\mathrm{t}_{\text {corr,LE }}=\mathrm{t}_{0, \mathrm{LE}}+\frac{\mathrm{w}}{\sqrt{\pi}} \Delta \mathrm{t}_{\mathrm{f}}+\left(\tau_{\mathrm{f}, \text { peak }}-\tau_{\mathrm{f}, \mathrm{LE}}\right)
$$

The second term in Eq. (12) accounts for the width correction as a result of the filter response time alone, and the last term accounts for the relative filter-group delays, $\tau_{\mathrm{f} \text {,peak }}$ for the peak-tagging stations and $\tau_{f, L E}$ for the leading-edge stations. We note that for narrowband SAW filters, depending on the choice of $\mathrm{w}_{1}$ and $\mathrm{w}_{2}$, the last term may dominate the correction. Finally, the TEC and $t_{0, L E}$ cannot be determined from DTOAs of different frequency channel pairs without a correction being applied. Indeed, barring the analysis given above, a lookup table would have to be developed from numerical simulations to correct for the nonlinear dependence of the leading-edge TOA on TEC.

In the analysis presented in this section, we have ignored the geomagnetic correction, and in the VHF, this approximation is justified by the fact that $f_{0}>f_{c}$. An iterative procedure can be used, however, to refine the analysis. First, an initial guess for $f_{c}$ and $\cos \beta$ (e.g., $\left.f_{c}=0\right)$ is used to determine the values of $t_{0}$ and a geolocation. Given an initial location, more accurate values for $\mathrm{f}_{\mathrm{c}}$ and $\cos \beta$ can be obtained, the fits recalculated, and new values obtained for $\mathrm{t}_{0}$ and the location. This iterative procedure does not supersede the analysis outlined above and should include the corrections provided in Eqs. (9) and (12) at each iteration.

\section{Verifying the Analytic Results and Correction Procedures}

As an example of the TOA corrections that might be necessary in a mixed network of leading-edge and peak-picking stations, we examine the simple case of a single impulse, $\mathrm{w}_{1}=1 / 4$, and $\mathrm{w}_{2}=3 / 4$, with a leading-edge bandwidth of $\mathrm{B}_{\mathrm{FWHM}}=3 \mathrm{MHz}$ and a peakpicking bandwidth of $\mathrm{B}_{\mathrm{FWHM}}=4.24 \mathrm{MHz}$ and find from Eq. (12) that $\mathrm{t}_{\text {corr,LE }}-\mathrm{t}_{0, \mathrm{LE}}=-149 \mathrm{~ns}$. The resulting correction is negative and indicates that we must reduce the leading-edge TOAs in order to use them in the location calculations performed with the mixed network. This result stems from the fact that the filter group delays are larger for the smaller bandwidth leading edge stations. We emphasize that the theoretical group delay dependence on bandwidth (Eq. 3) may not apply to all SAW filters and that the measured results should be used in Eq. (12) instead.

A numerical simulation that supports our analytic results is shown in Figs. 1a and $1 \mathrm{~b}$ where the detected signal in the linear amplitude domain (normalized to the peak amplitude) and computed TOA for exactly the same cases (i.e., source pulse and range to the respective stations) are shown for a leading-edge station and a peak-picking station, respectively. The equivalent log power plots are shown as Figs. $2 \mathrm{a}$ and $2 \mathrm{~b}$. Both stations are tuned to a central frequency of $50 \mathrm{MHz}$ and have an FWHM bandwidth of $3 \mathrm{MHz}$ for the leading-edge station and 4.24 MHz for the peak-picking station. The impulse used in the simulation is such that the rise time of the signal is much less than the filter response time, $\Delta \mathrm{t}_{\mathrm{f}}$. The TEC was set to zero. The amplitude points used in the leading-edge analysis are those noted above. The TOA in each case is referenced to the original source pulse initiation minus the vacuum travel time to the sensor and for the leading-edge and peak-picker stations is found to be $\mathrm{TOA}=7.951$ and $7.801 \mu \mathrm{s}$, respectively, for a 
difference of $-150 \mathrm{~ns}$. The negative sign indicates that the peak-picker station arrived first so that the leading-edge arrival time must be reduced to match the peak-picker station. These results are in agreement with the analytic calculations. Note that the filter group delays differ in each case (different bandwidths for each of the stations). The peak of the pulse for the case of the leading-edge station arrives at $~ 8.21 \mu \mathrm{s}$ but that of the peakpicker station arrives at $\sim 7.8 \mu \mathrm{s}$. The difference in these two peak times ( 410 ns) is consistent with the difference in group delays derivable from Eq. 3 for each station.

Another confirmation of our analysis lies in the work performed in Kim and Eng (1995). For the $w_{1}=1 / 3$ and $w_{2}=2 / 3$ analysis in their report, Kim and Eng derive a width correction coefficient ( $=\mathrm{w}$ in our notation), substantiated with actual laboratory test results, of $\mathrm{w}_{\text {Kim\&Eng }}=2.919$. Substituting into our Eq. 6 or Eq. 8, we derive a value that is exactly a factor of 2 less than that defined in Kim and Eng and used in Pongratz (2005 and 2007), i.e., $\mathrm{w}=1.4595$. However, the temporal width used in their analyses is defined to be exactly a factor of two less than in ours. Thus, we are in complete agreement.

The previous example $t_{0, \mathrm{LE}}$ was obtained for a TEC $=0$. We now examine the TEC determination procedure outlined in the previous section for the signal regime where only a single impulse arrives at the detector and verify that both $\mathrm{t}_{0, \mathrm{LE}}$ and $\mathrm{t}_{\text {corr,LE }}$ are correctly represented by Eq. 11, with $X=0$, and Eq. 12, respectively. The case of determining the TEC and infinite-frequency TOA from the raw leading-edge TOAs (without any correction) is also treated for comparison. Six frequency channels were used in this analysis; namely, $\mathrm{f}_{0}=40 \mathrm{MHz}, 50 \mathrm{MHz}, 70 \mathrm{MHz}, 110 \mathrm{MHz}, 120 \mathrm{MHz}$, and $130 \mathrm{MHz}$. The constants $\mathrm{w}_{1}$ and $\mathrm{w}_{2}$ were set to $1 / 4$ and $3 / 4$, respectively. The bandwidths of the six receivers were taken to be the same with a value of $\mathrm{B}_{\mathrm{FWHM}}=2 \mathrm{MHz}$ (note that the group delays for all channels will be the same). The channel trigger level was set to $12 \mathrm{~dB}$. The TEC values specified as input to the simulations were TEC $=10,20,30$, and $100 \times 10^{16} \mathrm{~m}^{-2}$, but the signal-to-noise ratios (SNRs) were grouped into three categories corresponding to high, medium, and low. The actual values varied from channel to channel, depending on ionospheric dispersion and the specified noise floor. For simplicity, our analysis was performed for the ordinary mode only, with $\mathrm{f}_{\mathrm{c}}=1.4 \mathrm{MHz}$ and $\cos \beta=1$. Results of our analysis are provided in Tables 1 and 2 . The first table lists $\mathrm{t}_{0}$ obtained from the peak-picking algorithm (see, e.g., Fig. 1b) followed by the difference in $t_{0}$ between the leading-edge algorithm (see, e.g., Fig. 1a) and the peak-picking algorithm obtained in three ways; namely, 1) from a linear fit to the corrected TOAs (Eq. 8) vs. $1 /\left[\mathrm{f}_{0}+\mathrm{f}_{\mathrm{c}} \cos \beta\right]^{2}$, (2) from a linear fit to the raw TOAs vs. $1 /\left[\mathrm{f}_{0}+\mathrm{f}_{\mathrm{c}} \cos \beta\right]^{2}$, and (3) from a nonlinear fit Eq. 11 of the raw TOAs vs. $1 /\left[\mathrm{f}_{0}+\mathrm{f}_{\mathrm{c}} \cos \beta\right]^{2}$. Each of the infinite frequency arrival times was then corrected according to Eq. 9 for method (1) and Eq. 12 for methods (2) and (3). The first correction is zero in our case because the group delays were assumed to be the same for all channels and for both peak-picker and leading-edge stations. The corresponding range of SNRs achieved across the six channels for a given TEC are listed in the final columns of Tables 1 and 2. As an example, Figs. $3 a-3 c$ show plots of the leading-edge corrected TOAs, the leading-edge raw TOAs, and the peak-selected TOAs, respectively, vs. $1 /\left[\mathrm{f}_{0}+\mathrm{f}_{\mathrm{c}} \cos \beta\right]^{2}$, along with the linear (3a and 
3c) or nonlinear (3b) fits for the simulated case of TEC $=20$ and low SNR. The corresponding values of $t_{0}$ and TEC obtained from the fits are also shown in these figures.

Assuming that the peak-selected $t_{0}$ serves as the standard, then good performance is indicated by a minimum in the differences listed in Table 1 . For the majority of cases, we find that the nonlinear fit to the raw TOAs performs best but not significantly better than the linear fit to corrected TOAs. In one case (TEC $=100, \mathrm{f}_{0}=40 \mathrm{MHz}$, low SNR), a leading-edge TOA was not found because the $\mathrm{w}_{1}$ point was in the noise (the value was set to a fixed offset relative to the trigger time) and a nonlinear fit, including that point, could not be determined. Instead, a nonlinear fit was made to the four highest frequency channels, and the resulting derived $t_{0}$ was found to be significantly different from $t_{0 \text {,peak }}$. In this one case, the nonlinear fit performed poorly compared to the linear fit to corrected TOAs. The linear fits to the raw TOAs performed poorly at the higher TECs (with the one exception being the very last entry, which we deem to be fortuitous) as expected and can be used as a standard of comparison.

Table 2 lists the differences between the derived TECs (from each of the four methods outlined for the $t_{0}$ determinations) and the actual specified values. The results are basically the same; namely, the nonlinear fits to raw TOAs perform to about the same fidelity as the linear fits to corrected TOAs, but the linear fits to raw TOAs serve as a standard for poor performance. With a few exceptions, the peak-picker algorithm performed best, as might be expected.

\section{Conclusions}

The corrections necessary to align the leading-edge and peak-picking infinite frequency times-of-arrival $\mathrm{t}_{0, \mathrm{LE}}$ and $\mathrm{t}_{0 \text {,peak }}$ were derived for arbitrary amplitude pairs used by the leading-edge time-tagging method. When the individual frequency channel TOAs are corrected for width effects and a $\mathrm{t}_{0, \mathrm{LE}}$ is then derived from a linear fit to the corrected TOAs vs. $1 /\left[\mathrm{f}_{0} \pm \mathrm{f}_{\mathrm{c}} \cos \beta\right]^{2}$, Eq. 9 provides the correction for alignment. If a linear or nonlinear fit to the raw leading edge TOAs vs. $1 /\left[\mathrm{f}_{0} \pm \mathrm{f}_{\mathrm{c}} \cos \beta\right]^{2}$ is used to determine $\mathrm{t}_{0, \mathrm{LE}}$ as outlined above, then Eq. 12 provides the necessary correction for alignment. The latter equation is also appropriate when a lookup table is used to derive $\mathrm{t}_{0, \mathrm{LE}}$. Note that the technique of nonlinear fitting should only be used when a lookup table that takes into account additional effects, as noted for example by Massey, Blake, and Hoard, 1990, is not available.

One important detail not fully treated in this study involves the effect of the geomagnetic field on the ionospheric delay. Most receivers are designed to accept only one or the other mode (ordinary or extraordinary) in order to avoid the time-tagging uncertainties associated with the beating of the two modes within the filter bandwidth. With only one mode, either the + or - sign should be used in the fitting algorithms. In order to proceed, however, it is also necessary to know the values of $f_{c}$ and $\cos \beta$, which can only be determined precisely after geolocation. Thus, an iterative approach is necessary in which an initial guess is provided for $f_{c}$ and $\cos \beta$. Fortunately, in the VHF, $\mathrm{f}_{0}$ is large compared to the gyrofrequency, and the iterative approach works well. A more 
quantitative analysis of geomagnetic corrections to the ionospheric dispersion in the context of time-tagging accuracy is left for future work.

The analytic results and supporting simulations presented in this report indicate that for a single impulse (without multipathing or reflections), the linear fit to corrected TOAs and the nonlinear fit to uncorrected TOAs provide approximately the same accuracy although the nonlinear fitting algorithm seemed to perform a little better in the majority of cases. Deriving an infinite-frequency time-of-arrival or TEC from the raw leadingedge TOAs leads to larger errors that increase with TEC, and this method therefore forms the standard for poor performance. We also note that the nonlinear fitting routine requires at least four points (or frequency channels) to arrive at a convergent solution where three parameters are allowed to vary.

Three signal regimes were defined in which the leading-edge TOAs could either be corrected to align with the peak-picking algorithm or would potentially perform better than the peak-picker. The first corresponds to a single impulse (the one studied in this report in some detail). In this case, we expect the peak-picker to set the standard. The second regime deals with the rapid arrival of additional impulsive signals following the signal of interest so that the leading edge of the first signal is now distorted. In this case, the time delay of the follow-on signals is less than the 1/e half width of the signal, i.e., $t_{\text {delay }}<\Delta t_{\text {total }}$. We expect the peak-picker in this case to again set the standard and the corrections derived in this report for the leading edge to apply. A thorough study for the range of scenarios of interest is recommended to verify this conclusion. The third regime is defined by signals that arrive so that $t_{\text {delay }}>\Delta t_{\text {total }}$, assuming that the amplitudes of the secondary signals are less than or not much greater than the first impulse. In this case, the leading edge of the detected signal is undistorted and associated solely with the arrival of the signal of interest. The leading-edge algorithm is expected to outperform the peakpicker in these cases, but a detailed study is required to quantify the performance for the gamut of possible scenarios.

Finally, the results obtained in this report provide a means for correcting the leadingedge arrival times to align with the peak-picker arrival times, but they should not be implemented until a detailed study is performed with actual data obtained with the specific location network of concern. Equally important is the need to reject any algorithms or corrections that are based entirely on data analysis. Not only are the data unlikely to cover the entire parameter space of interest, but the lack of theoretical understanding and a basis for making recommended changes is dangerous and can lead to significant unanticipated errors. For the WSRP, the detailed report and study performed by Massey, Blake, and Hoard, 1990, still forms the standard, but the arrival times obtained after corrections from the table are applied should still be adjusted according to Eq. 12 to align them with the peak-picker platforms. 


\section{References}

Kim, Y.S., and R. Eng, "Time-of-Arrival Prediction Model for Transionospheric EMP," IEEE Transaction on Aerospace and Electronic Systems 31, No.1, 409-413 (1995).

Massey, R.S., J. Blake, and D. Hoard, "A Lookup Table Approach for the G/AIT Algorithm,” LA-CP-90-205 (1990).

Massey, R.S., "Impulse Response Function for a Transionospheric Signal through a Gaussian Filter," Radio Science 18, No. 4, pp. 643-647 (1993).

Pongratz, M.B., “WSRP Bias Calibration Studies (U),” LA-CP-07-0232 (2007).

Pongratz, M.B., D.M. Suszcynsky, and P.S. McLachlan, "EMP Sensor Geolocation Studies (U)," LA-CP-07-0232 (2007).

Pongratz, M.B., "A Dual Band Algorithm to Remove Ionospheric Dispersion (U)," Los Alamos National Laboratory Report No. LA-CP-05-0139 (2005). 


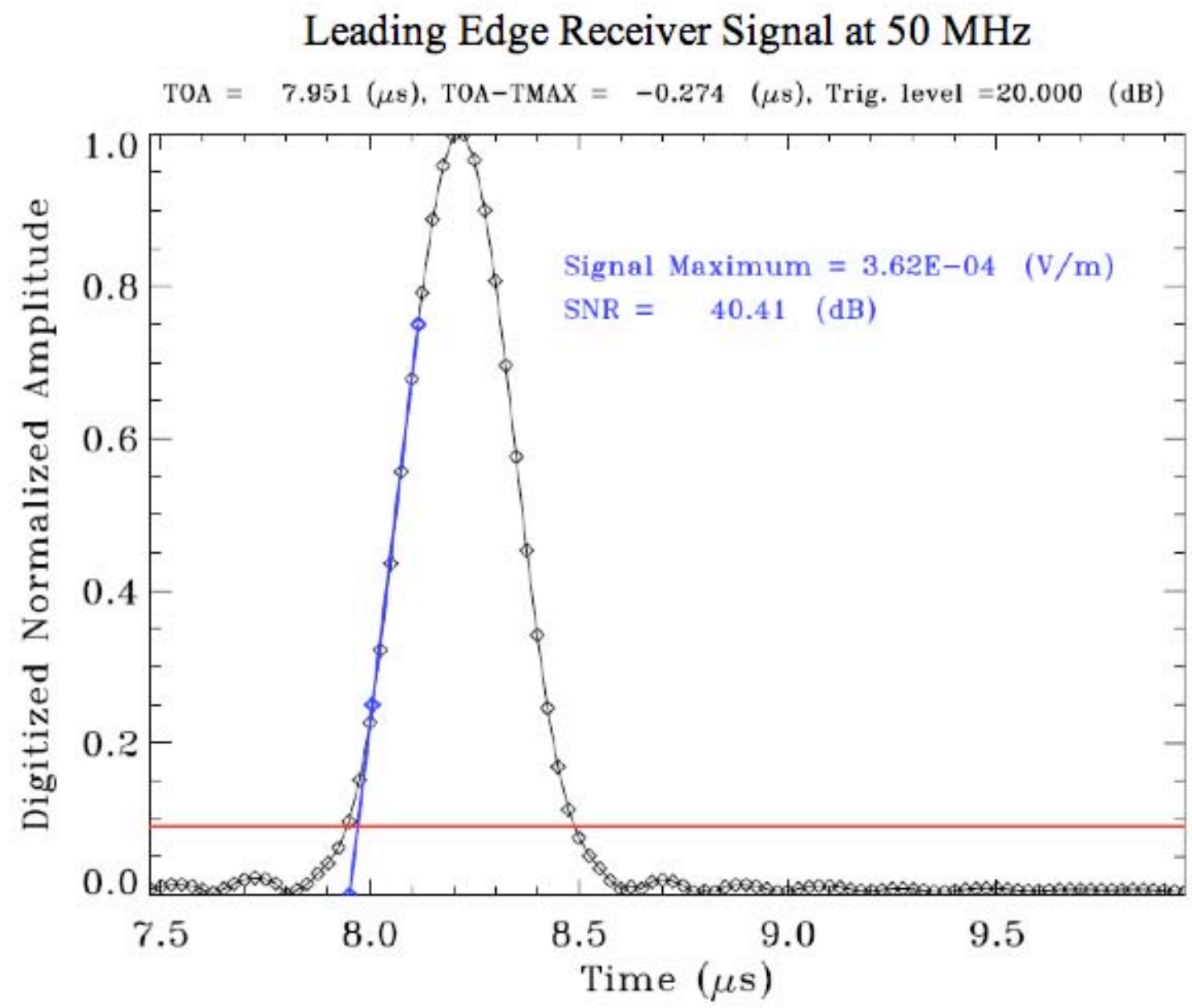

Figure 1a. Leading-edge time-tagging of an impulsive signal. The signal strength (in normalized amplitude units) detected by a receiver tuned to $50 \mathrm{MHz}$ and having a $3 \mathrm{MHz}$ full width at half maximum (FWHM) bandwidth is plotted as a function of time. The TEC was set to zero in this case so that the filter response $\Delta \mathrm{t}_{\mathrm{f}}$ defines the pulse width in this plot. The red line indicates the relative amplitude level at which the detector triggered. The blue line represents the linear fit to the $\mathrm{w}_{1}(=1 / 4 \mathrm{peak}$ amplitude, lower blue diamond on curve $)$ and $\mathrm{w}_{2}(=3 / 4$ peak amplitude, upper blue diamond on curve $)$ points and its extrapolation to zero signal level at the TOA. The TOA is referenced to the original source pulse initiation minus the vacuum travel time to the sensor and for this channel, the TOA $=7.951 \mu \mathrm{s}$. 


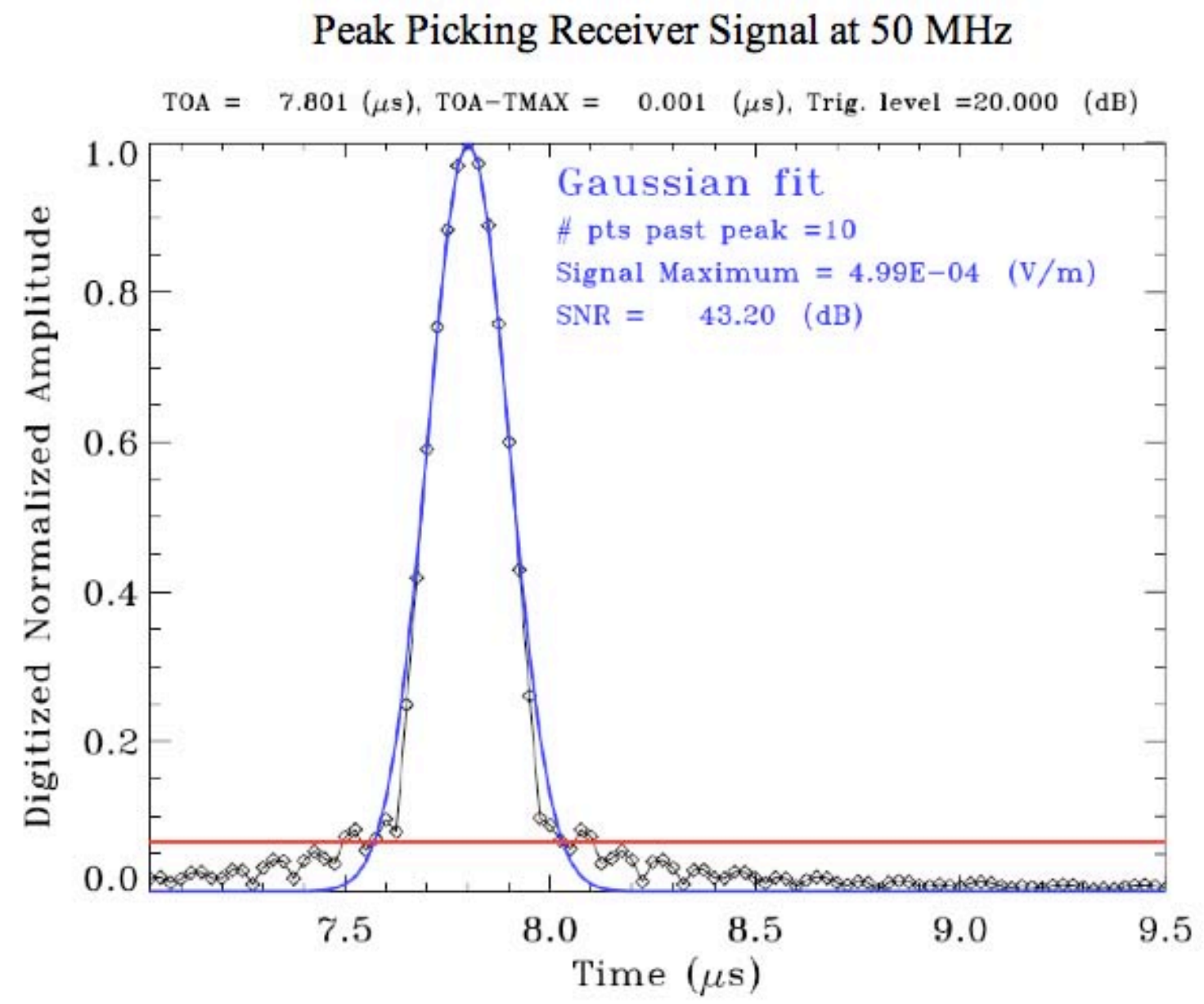

Figure 1b. Peak-picker time-tagging of an impulsive signal. The signal strength (in normalized amplitude units) detected by a receiver tuned to $50 \mathrm{MHz}$ and having a 4.24 MHz FWHM bandwidth is plotted as a function of time. As in Fig. 1a, the TEC was set to zero so that the filter response $\Delta \mathrm{t}_{\mathrm{f}}$ defines the pulse width in this plot. The red line indicates the relative amplitude level at which the detector triggered. The blue line is the equivalent Gaussian representation in the linear domain of a parabolic fit in log power to points around the signal maximum (see Fig. 2b). The fit provides a better estimate of the peak TOA for a digitized signal. The TOA is referenced to the original source pulse initiation minus the vacuum travel time to the sensor, and for this channel $\mathrm{TOA}=7.801 \mu \mathrm{s}$. 


\section{Leading Edge Receiver Signal at $50 \mathrm{MHz}$}

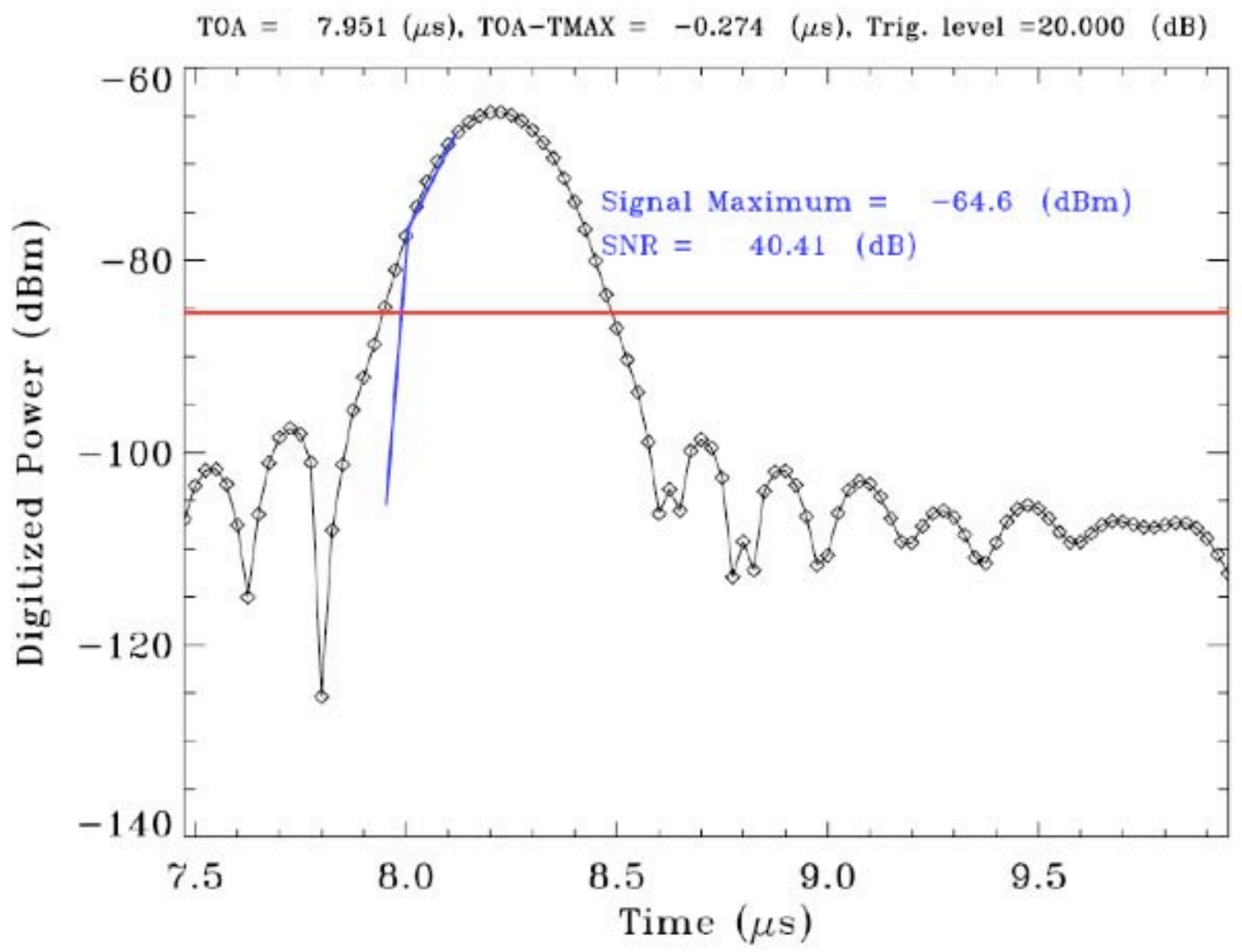

Figure 2a. Leading-edge time-tagging of an impulsive signal. The signal power (in $\mathrm{dBm}$ ) detected by a receiver tuned to $50 \mathrm{MHz}$ and having a $3 \mathrm{MHz}$ FWHM bandwidth is plotted as a function of time. The red line indicates the power level at which the detector triggered. The blue line represents the linear fit (in amplitude, not log power) to the $\mathrm{w}_{1}(=1 / 4$ peak amplitude, not power $)$ and $\mathrm{w}_{2}(=3 / 4$ peak amplitude, not power $)$ points and its extrapolation to the mean noise level. The TOA is referenced to the original source pulse initiation minus the vacuum travel time to the sensor, and for this channel TOA $=7.951 \mu \mathrm{s}$. 


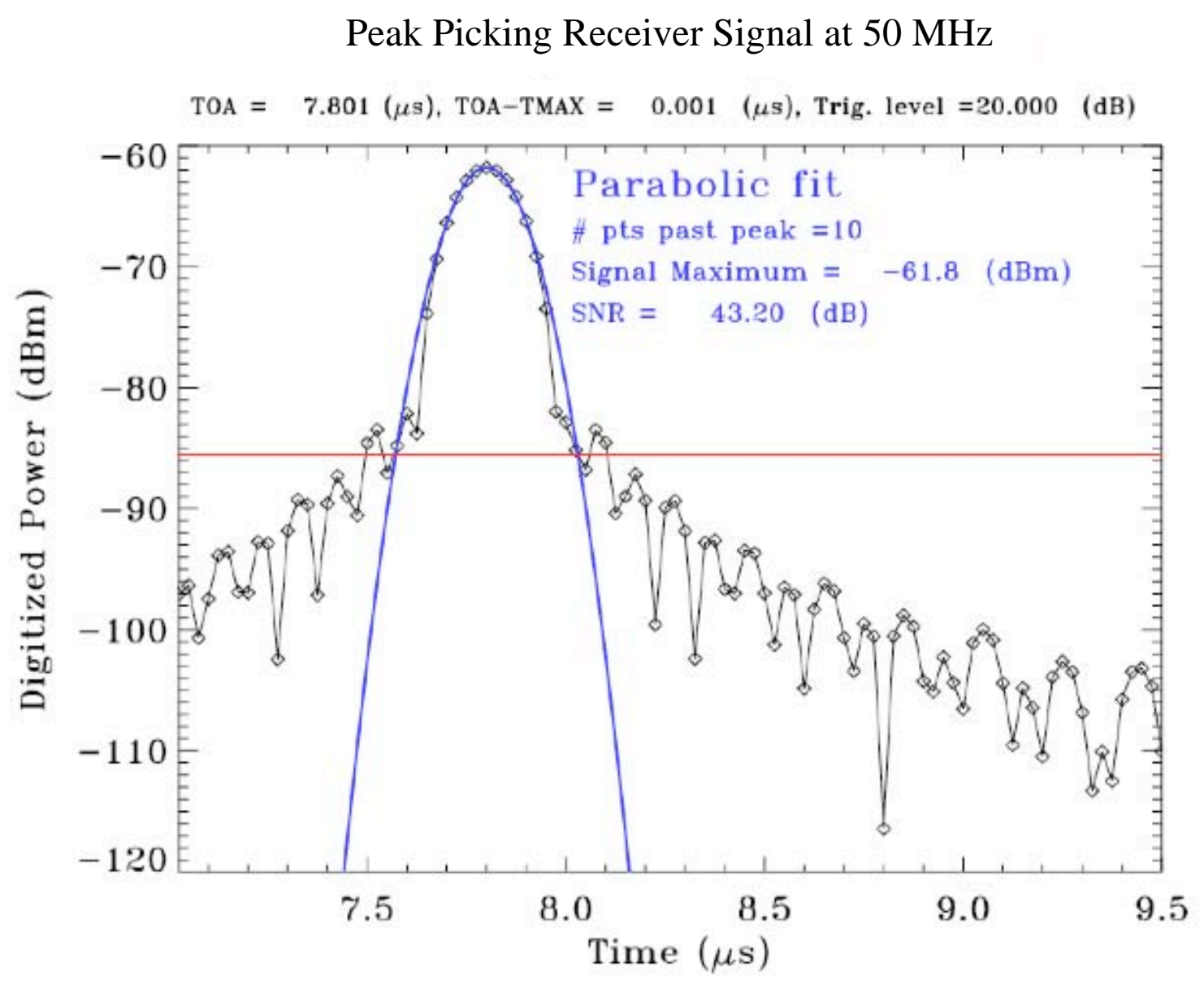

Figure 2b. Peak-picker time-tagging of an impulsive signal. The signal power (in $\mathrm{dBm}$ ) detected by a receiver tuned to $50 \mathrm{MHz}$ and having a $4.24 \mathrm{MHz}$ FWHM bandwidth is plotted as a function of time. The red line indicates the power level at which the detector triggered. The blue line is a parabolic fit to points around the signal maximum. The fit provides a better estimate of the peak TOA for a digitized signal. The TOA is referenced to the original source pulse initiation minus the vacuum travel time to the sensor, and for this channel TOA $=7.801 \mu \mathrm{s}$. 


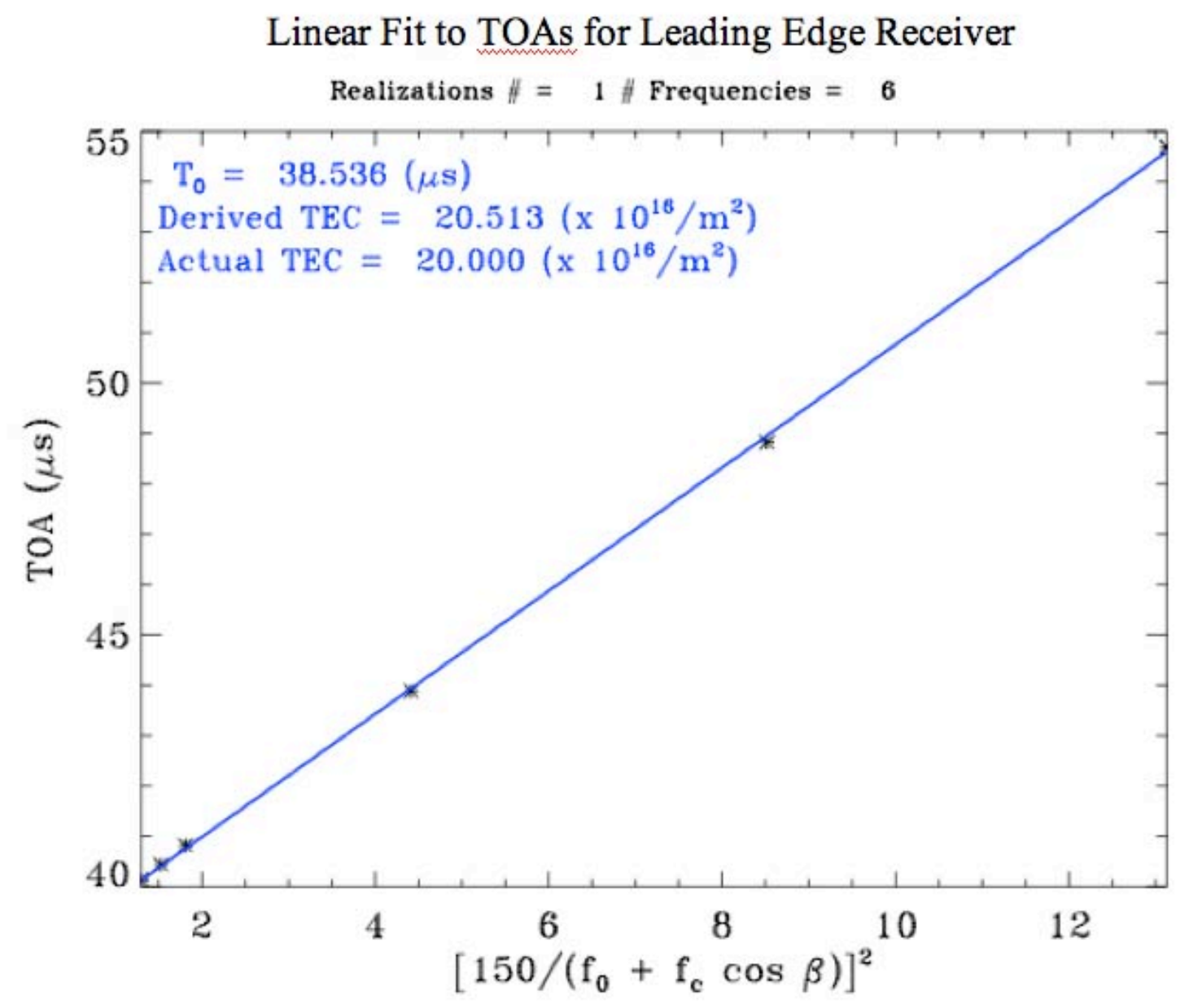

Figure 3a. Fit to corrected leading-edge TOAs. The infinite-frequency TOA $\mathrm{T}_{0}$ and the TEC were derived from a linear fit to the leading-edge, corrected TOAs (plotted as stars, see Eq. 8) vs. $1 /\left[\mathrm{f}_{0}+\mathrm{f}_{\mathrm{c}} \cos \beta\right]^{2}$ for six frequency channels (see text) with $\mathrm{f}_{\mathrm{c}}=1.4 \mathrm{MHz}$ and $\cos \beta=1$ and for the simulated case with a TEC $=20 \times 10^{16} / \mathrm{m}^{2}$ and low signal-tonoise as defined in Tables 1 and 2 . The blue line corresponds to the fit, and the blue labels provide the derived values of $\mathrm{T}_{0}$ and TEC along with the actual TEC. Note that the TOAs are referenced to the original source pulse initiation minus the vacuum travel time to the sensor. 


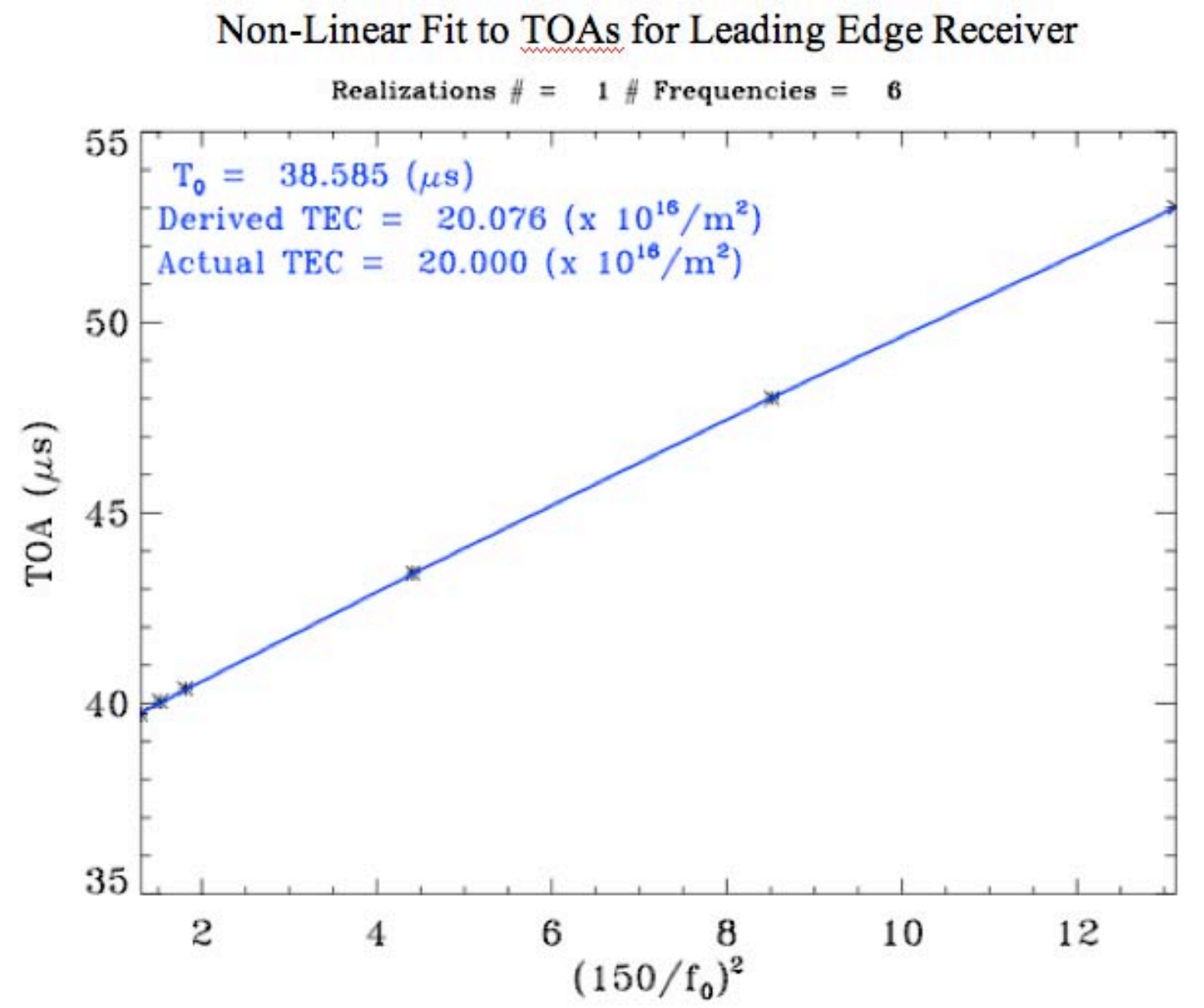

Figure 3b. Nonlinear Fit to Raw Leading Edge TOAs. The infinite-frequency TOA $\mathrm{T}_{0}$ and the TEC were derived from a nonlinear fit to the leading-edge, raw TOAs (plotted as stars, see Eq. 11) vs. $1 /\left[\mathrm{f}_{0}+\mathrm{f}_{\mathrm{c}} \cos \beta\right]^{2}$ for six frequency channels (see text) with $\mathrm{f}_{\mathrm{c}}=1.4 \mathrm{MHz}$ and $\cos \beta=1$ and for the simulated case with a TEC $=20 \times 10^{16} / \mathrm{m}^{2}$ and low signal-to-noise as defined in Tables 1 and 2 . The blue line corresponds to the fit, and the blue labels provide the derived values of $\mathrm{T}_{0}$ and TEC along with the actual TEC. Note that the TOAs are referenced to the original source pulse initiation minus the vacuum travel time to the sensor. 


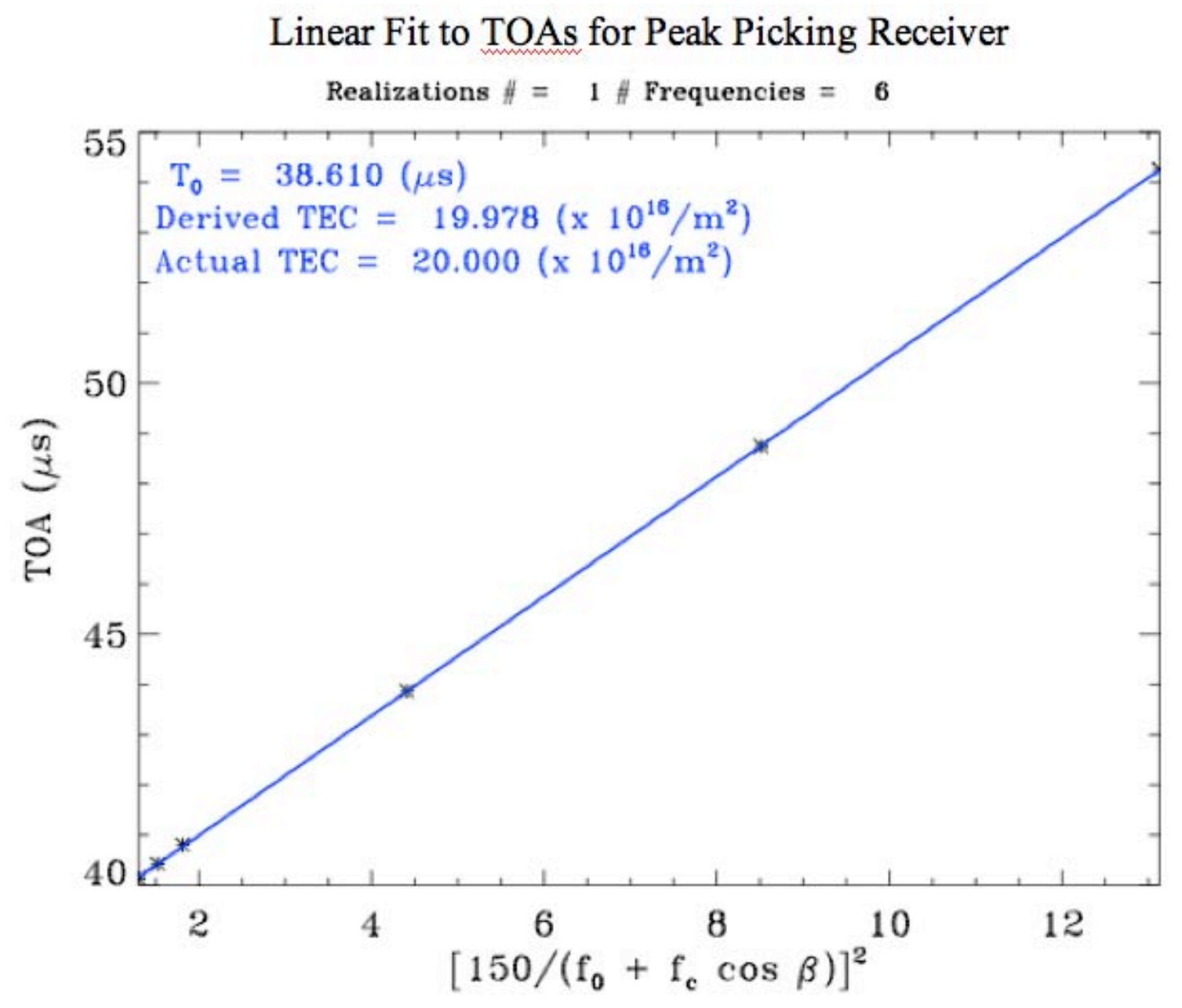

Figure 3c. Fit to peak-selected TOAs. The infinite-frequency TOA $\mathrm{T}_{0}$ and the TEC were derived from a linear fit to the peak-selected TOAs (plotted as stars) vs. $1 /\left[\mathrm{f}_{0}+\mathrm{f}_{\mathrm{c}} \cos \beta\right]^{2}$ for six frequency channels (see text) with $\mathrm{f}_{\mathrm{c}}=1.4 \mathrm{MHz}$ and $\cos \beta=1$ and for the simulated case with a TEC $=20 \times 10^{16} / \mathrm{m}^{2}$ and low signal-to-noise as defined in Tables 1 and 2. The blue line corresponds to the fit, and the blue labels provide the derived values of $\mathrm{T}_{0}$ and TEC along with the actual TEC. Note that the TOAs are referenced to the original source pulse initiation minus the vacuum travel time to the sensor. 
Table 1. Results of Leading-Edge Study for $T_{0}$. The actual TECs listed in column one are the values specified in the simulations. The results listed in column two are the peak arrival times $\mathrm{T}_{0 \text {,peak }}$ of the signal at the detector relative to an arbitrary but TECdependent start time. A $\mathrm{T}_{0}$ was calculated from a linear fit to width-corrected leadingedge TOAs vs. $1 /\left[\mathrm{f}_{0}+\mathrm{f}_{\mathrm{c}} \cos \beta\right]^{2}$ (see text) and then subtracted from $\mathrm{T}_{0 \text {,peak }}$ to obtain the values in column three. The results listed in both columns four and five use the raw leading edge TOAs for each channel to obtain a $\mathrm{T}_{0}$ (from linear and nonlinear fits, respectively, as noted in the text) that is then corrected for the bandwidth of the receiver according to Eq. (12), and the result is subtracted from $\mathrm{T}_{0 \text {,peak. }}$. The $\mathrm{SNR}$ column lists the range of signal-to-noise ratios achieved across the six frequency channels used in the study, and results are presented for nominally high, medium, and low SNRs.

\begin{tabular}{|c|c|c|c|c|c|}
\hline $\begin{array}{c}\text { TEC } \\
\left(10^{16} / \mathrm{m}^{2}\right)\end{array}$ & $\begin{array}{l}\text { Peak Picker } \\
\mathbf{T}_{0, \text { peak }}(\mu \mathrm{s})\end{array}$ & $\begin{array}{l}\mathbf{T}_{0, \text { peak }}-\mathbf{T}_{\mathbf{0 , L E}}(\mu \mathrm{s}) \\
\text { Width Corrected }\end{array}$ & $\begin{array}{c}T_{0, p e a k}-T_{0, L E}(\mu s) \\
\text { Raw TOAs }\end{array}$ & $\begin{array}{c}\mathbf{T}_{0, \text { peak }}-\mathbf{T}_{0, \text { LE }}(\mu \mathrm{s}) \\
\text { NonLinear Fit }\end{array}$ & $\begin{array}{l}\text { SNR } \\
\text { (dB) }\end{array}$ \\
\hline 10 & 36.790 & .019 & -.040 & .006 & $22-32$ \\
\hline 20 & 38.602 & .031 & -.143 & . 020 & $21-30$ \\
\hline 30 & 48.412 & -.006 & -.228 & .014 & $21-29$ \\
\hline 100 & 116.891 & -.730 & -.869 & -.248 & $21-28$ \\
\hline 10 & 36.787 & .027 & -.026 & .003 & 18-27 \\
\hline 20 & 38.606 & .043 & -.140 & .028 & $16-25$ \\
\hline 30 & 48.419 & -.022 & -.219 & .025 & $16-24$ \\
\hline 100 & 117.328 & -.547 & -.452 & 212 & $16-23$ \\
\hline 10 & 36.781 & .032 & -.005 & .001 & $16-22$ \\
\hline 20 & 38.610 & .074 & -.128 & .025 & $16-20$ \\
\hline 30 & 48.442 & -.047 & -.185 & .060 & 13-19 \\
\hline $100 * *$ & 116.881 & -.059 & .011 & .233 & $13-17$ \\
\hline
\end{tabular}

*** No $\mathrm{w}_{1}$ point was found on the leading edge for the lowest frequency channel. 
Table 2. Results of Leading-Edge Study for TEC. The actual TECs listed in column one are the values specified in the simulations. The TECs used to obtain the differences listed in the various columns were determined from the coefficients of linear or nonlinear fits to the TOAs (from leading edge or peak methods as noted) vs. $1 /\left[\mathrm{f}_{0}+\mathrm{f}_{\mathrm{c}} \cos \beta\right]^{2}$ as described in the text. The SNR column lists the range of signal-to-noise ratios achieved across the six frequency channels used in the study, and results are presented for nominally high, medium, and low SNRs.

\begin{tabular}{|c|c|c|c|c|c|}
\hline $\begin{array}{l}\text { Actual TEC } \\
\left(10^{16} / \mathrm{m}^{2}\right)\end{array}$ & $\begin{array}{l}\text { Peak Picker } \\
\text { TEC-Actual } \\
\left(10^{16} / \mathrm{m}^{2}\right)\end{array}$ & $\begin{array}{c}\text { Width Corrected } \\
\text { LE } \\
\text { TEC-Actual } \\
\left(10^{16} / \mathrm{m}^{2}\right)\end{array}$ & $\begin{array}{c}\text { Raw LE } \\
\text { TEC-Actual } \\
\left(10^{16} / \mathrm{m}^{2}\right)\end{array}$ & $\begin{array}{c}\text { Nonlinear Fit LE } \\
\text { TEC-Actual } \\
\left(\mathbf{1 0}^{16} / \mathbf{m}^{2}\right)\end{array}$ & $\begin{array}{l}\text { SNR } \\
\text { (dB) }\end{array}$ \\
\hline $\begin{array}{c}10 \\
20 \\
30 \\
100\end{array}$ & $\begin{array}{l}.028 \\
-.003 \\
-.066 \\
.715\end{array}$ & $\begin{array}{c}.083 \\
.194 \\
.021 \\
-4.614\end{array}$ & $\begin{array}{l}-.408 \\
-1.254 \\
-2.119 \\
-7.769\end{array}$ & $\begin{array}{l}.051 \\
.049 \\
-.125 \\
-2.026\end{array}$ & $\begin{array}{l}22-32 \\
21-30 \\
21-29 \\
21-28\end{array}$ \\
\hline $\begin{array}{c}10 \\
20 \\
30 \\
100\end{array}$ & $\begin{array}{l}.038 \\
-.010 \\
-.104 \\
-2.039\end{array}$ & $\begin{array}{l}.127 \\
.290 \\
.014 \\
-6.535\end{array}$ & $\begin{array}{l}-0.341 \\
-1.222 \\
-2.090 \\
-7.943\end{array}$ & $\begin{array}{l}-.113 \\
.095 \\
-.122 \\
-1.870\end{array}$ & $\begin{array}{l}18-27 \\
16-25 \\
16-24 \\
16-23\end{array}$ \\
\hline $\begin{array}{c}10 \\
20 \\
30 \\
100 * * \\
\end{array}$ & $\begin{array}{l}.056 \\
-.022 \\
-.191 \\
.717 \\
\end{array}$ & $\begin{array}{l}.166 \\
.513 \\
-.010 \\
-.913 \\
\end{array}$ & $\begin{array}{l}-.260 \\
-1.145 \\
-2.029 \\
-2.334 \\
\end{array}$ & $\begin{array}{l}-.181 \\
.076 \\
-.079 \\
8.150 \\
\end{array}$ & $\begin{array}{l}16-22 \\
16-20 \\
13-19 \\
13-17 \\
\end{array}$ \\
\hline
\end{tabular}

${ }^{* * *}$ No $\mathrm{w}_{1}$ point was found on the leading edge for the lowest frequency channel. 
This report has been reproduced directly from the best available copy. It is available electronically on the Web (http://www.doe.gov/bridge).

Copies are available for sale to U.S. Department of Energy employees and contractors from:

Office of Scientific and Technical Information

P.O. Box 62

Oak Ridge, TN 37831

(865) 576-8401

Copies are available for sale to the public from: National Technical Information Service

U.S. Department of Commerce

5285 Port Royal Road

Springfield, VA 22161

(800) 553-6847 
- Los Alamos NATIONAL LABORATORY

EST.1943 